\title{
Saturation Effects in Field-Oriented Induction Machines
}

ROBERT D. LORENZ, MEMBER, IEEE, AND DONALD W. NOVOTNY, FELLOW, IEEE

Abstract-The important effects of saturation are outlined, and a simple first-order piecewise linear representation of the saturation characteristic that explains these effects in a simple, easy to understand manner, is presented. Machine properties explored include saturation effects on available torque per stator ampere, practical limits on available torque and torque linearity, and a general analysis of the influence of parameter variations (detuning) in adjustable flux systems. An experimental technique for evaluating the $\boldsymbol{d}$-axis current level associated with peak torque per stator ampere in a saturated machine is also presented.

\section{INTRODUCTION}

M AIN FLUX saturation plays an important role in field-oriented induction machine drives. Of major significance are

- effects on available torque per ampere [1],

- effects on motor efficiency,

- practical limits on available torque and torque linearity [2], and

- parameter variations and detuning effects in adjustable flux systems.

The goal of this paper is to outline the important effects of saturation and to present a simple first-order piecewise linear representation of the saturation characteristic that explains these effects in a simple, easy to understand manner. The current-fed properties of induction machines and how these properties are employed when a field-oriented controller is used are first outlined (see [3] for a review of field-oriented control theory). The following section outlines a simple but demonstrably effective saturation model that allows direct noniterative estimation of machine properties, which are very close to those measured. The following sections then use the model to demonstrate and evaluate the effects of saturation on

- actual flux levels,

- maximum torque production,

- maximum torque per ampere squared,

- linearity of the torque response for detuned field-oriented machines.

The last section describes the details of an experimental

Paper IPCSD 89-31, approved by the Industrial Drives Committee of the IEEE Industry Applications Society for presentation at the 1987 IEEE Industry Applications Society Annual Meeting, Atlanta, GA, October 19-23. Manuscript released for publication July 26, 1989.

The authors are with the Department of Electrical and Computer Engineering, University of Wisconsin-Madison, 1415 Johnson Drive, Madison, W1 53706

IEEE Log Number 8932125.

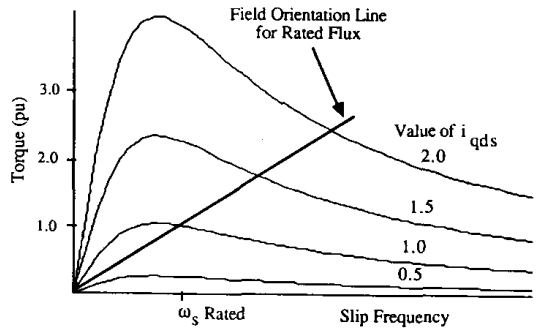

Fig. 1. Torque versus slip frequency characteristic of typical current-fed induction machine (no saturation).

technique to determine the flux-producing current level from laboratory test data.

The Constant Parameter Induction Machine Model

\section{General Model}

The general model of a current-fed induction machine is obtained by combining the general rotor voltage and flux equations with the general torque production equation as follows. (The terms are defined in Appendix I).

For rotor voltage:

$$
0=R_{r} \bar{i}_{q d r}+\left(p+j \omega_{s}\right) \bar{\lambda}_{q d r} .
$$

For rotor flux linkage:

$$
\bar{\lambda}_{q d r}=L_{m} \bar{i}_{q d s}+L_{r} \bar{i}_{q d r} .
$$

For torque production:

$$
\tilde{T}=\frac{3}{2} \frac{P}{2} \bar{\lambda}_{q d r} \times \bar{i}_{q d s}
$$

The resulting current controlled torque equation for steadystate operation is

$$
T=\frac{3}{2} \frac{P}{2} \frac{L_{m}^{2}}{L_{r}}\left|\bar{i}_{q d s}\right|^{2} \frac{\omega_{s} \tau_{r}}{1+\left(\omega_{s} \tau_{r}\right)^{2}} .
$$

From these equations the resulting current-fed induction machine torque versus slip frequency characteristic is readily calculated and is shown in Fig. 1.

For consideration of saturation the rotor flux is a critical variable. For the nonsaturating constant parameter machine, it can be calculated from the rotor voltage (1) and flux linkage equations (2) and may be written as

$$
\bar{\lambda}_{q d r}=L_{m} \bar{i}_{q d s} \frac{1}{1+j \omega_{s} \tau_{r}} .
$$




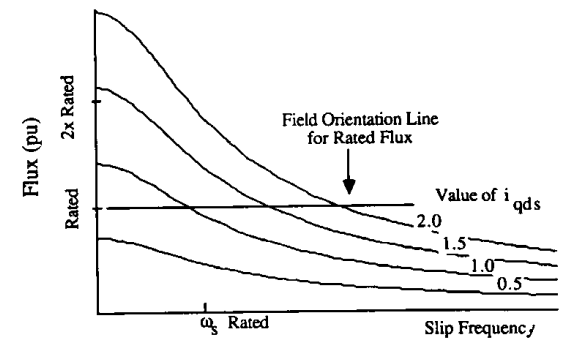

Fig. 2. Rotor flux versus slip frequency characteristic of current-fed induction machine (no saturation).

When plotted as in Fig. 2 this model displays the large increase in rotor flux that would occur if no saturation effects were present.

\section{Applying the Field-Oriented Control Constraints}

The field-oriented control constraint for orthogonality of flux and torque producing components of the stator current is

$$
\bar{\lambda}_{q d r}=-j \lambda_{d r}
$$

Solving both the flux linkage and the torque equation with this constraint provides the following slip calculation function (slip relation):

$$
\omega_{s}=\frac{1}{\tau_{r}} \frac{L_{m}}{\lambda_{d r}} i_{q s}
$$

equivalent torque production function:

$$
T=\frac{3}{2} \frac{P}{2} \frac{L_{m}}{L_{r}} \lambda_{d r} i_{q s},
$$

and rotor flux response function:

$$
\lambda_{d r}=L_{m} i_{d r} \frac{1}{1+p \tau_{r}},
$$

for field-oriented induction machines. These field-oriented constraint functions are shown as the straight lines (for steadystate at constant flux) in Figs. 1 and 2.

\section{A Piecewise Linear Saturation Model}

Experience and intuition suggest that large magnetizing currents will be required to create flux levels significantly larger than the nominal rated flux. Measurements on representative machines indicate that values in excess of 1.3 to 1.4 times rated flux will require magnetizing currents of the order of two times rated current.

Since such large magnetizing currents are clearly not useful in operation, a model that essentially limits the flux to a specific level, i.e., a flat flux versus current model, can be employed to demonstrate the primary effects of main flux saturation. Such a model gives very reasonable agreement with more exacting models of saturation [4]. For flux levels below this saturation flux, the magnetizing current can be taken as linear as shown in Fig. 3. Note that the "chord slope magnetizing inductance" also illustrated in the figure will vary inversely proportional to the $d$-axis current for this saturation model.

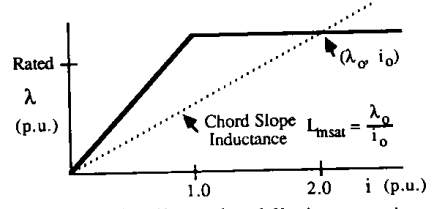

Fig. 3. Piecewise linear hard limit saturation model.

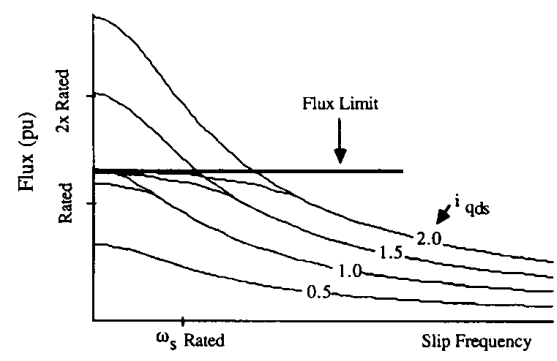

Fig. 4. Overlay of flux versus slip frequency characteristics for constan parameter and piecewise linear models for typical current-fed induction machine.

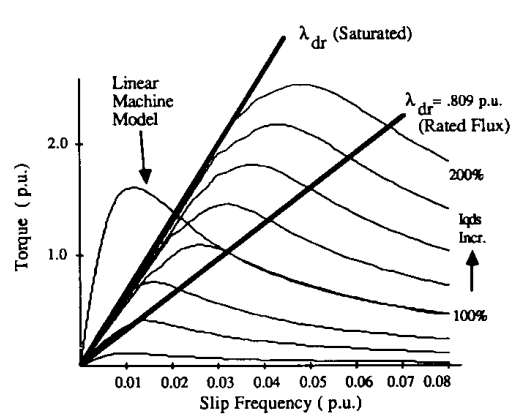

Fig. 5. Overlay versus slip frequency characteristics for constant parameter and piecewise linear models for typical current-fed induction machine.

\section{Machine Flux Characteristic with Saturation}

For the constant parameter (nonsaturating) machine, rotor flux is a function of slip frequency, stator current amplitude, and machine parameters as shown in (5). If one incorporates the flux limited saturation model of Fig. 3, the flux is limited to the saturation flux and the flux versus slip frequency characteristics will take on the form shown in Fig. 4. This figure shows the linear flux characteristic (Fig. 2) with the flux limit as an overlay. The actual saturated flux curves will follow some intermediate path in a smooth fashion joining the linear characteristic to the flux limit as illustrated.

\section{Machine Torque Characteristic with Saturation}

The saturation flux limit also has a major influence on the torque characteristics of Fig. 1. The nature of this effect is illustrated in Fig. 5. Since a constant flux operating line on this figure is a radial line through the origin (a "field orientation line"), the saturation flux limit line can be viewed as a limit line of the saturated torque characteristics as shown in Fig. 5. A torque production model conceptually based on the hard limit flux characteristic of Fig. 3 would follow the linear model torque curves for high slip frequencies and then abruptly switch to the straight-line saturated flux limit curve for small slip frequencies as illustrated by the rated current 


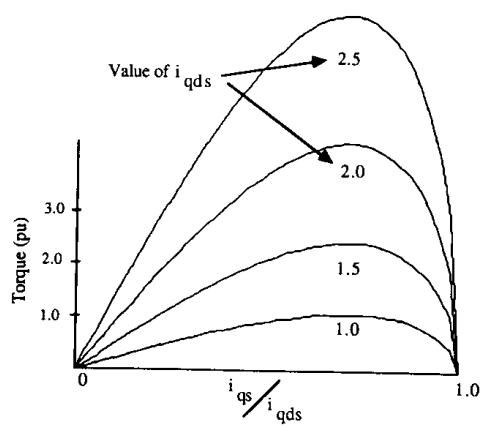

Fig. 6. Field-oriented machine torque production as function of percentage of stator current that torque command represents (no saturation).

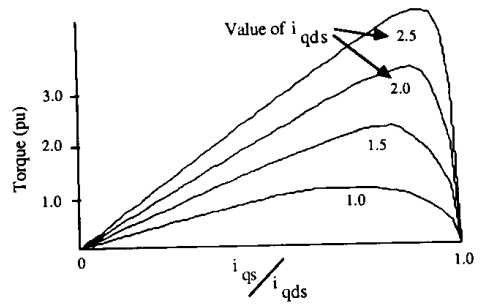

Fig. 7. Field-oriented machine torque production as function of percentage of stator current that the torque command represents (with saturation).

curve in Fig. 5. The actual saturated torque characteristics would depart somewhat from this conceptual model as shown in the figure.

The curves of Fig. 5 illustrate the large reduction in torque which occurs as a result of main flux saturation. The significant increase in the slip frequency for peak torque is also shown. Note that a field orientation line close to the saturation line exhibits nearly optimal torque per stator ampere over a wide range of torque levels in the saturated case. These issues are explored in more detail in the next section.

\section{Peak Torque Per Stator Ampere Investigation}

The torque production equation for a properly functioning field-oriented induction machine is given by (8). If the piecewise linear saturation model is applied to the rotor flux in this equation, it is possible to demonstrate some limiting issues for peak torque and alternate normalized measures such as peak torque per ampere or per ampere squared.

From (4) it is apparent that for a nonsaturating machine, the output torque will vary proportionally to the square of the stator current amplitude. For steady-state conditions Fig. 6 shows the torque as a function of the percentage of stator current that the torque command represents, as applied to the machine of Appendix I without saturation.

As expected, the torque envelope increases with the square of the current. For this case, the peak torque per stator ampere or per stator ampere squared always occurs at the value of $i_{q s}$ $=0.707 i_{q d s}$, or equivalently, where $i_{q s}=i_{d s}$.

Fig. 7 shows the same field-oriented machine plots, but using the piecewise linear saturation model to estimate the actual rotor flux. These results are dramatically different from Fig. 6 for the nonsaturating machine. The peaks of Fig. 7 no longer occur at a constant percentage of $i_{q s} / i_{q d s}$. As shown,

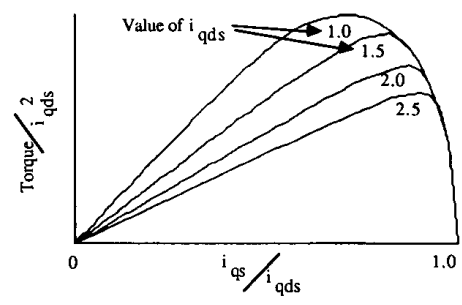

Fig. 8. Field-oriented machine torque production (normalized by division by current squared) as function of percentage of stator current that the torque command represents (with saturation).

the percentage of torque producing current increases as stator current amplitude increases. This is consistent with the fact that little flux increase will occur with increases in the magnetizing component $i_{d s}$; thus the peak torque will be best achieved by putting most of the increased current level into the torque command.

The data of Fig. 7 can also be normalized by division by either stator current amplitude or stator current amplitude squared. Fig. 8 shows the data normalized by stator current amplitude squared. This figure demonstrates how saturation reduces the torque response from the squared relationship to stator current, which held for nonsaturated conditions. It is readily apparent that the best condition is obtained just prior to the beginning of saturation. The torque per ampere squared is lower for any of the regions of saturated operation.

\section{Parameter Sensitivity for Detuned Operation}

If the gain of the slip calculator (i.e., the rotor time constant) is incorrect, the decoupling of flux and torque is disturbed and the field-oriented controller is said to be detuned. The sensitivity of a given drive to various amounts of gain error depends on the machine parameters and hence on the level of magnetic saturation of the main flux paths in the machine. To illustrate the influence of saturation, a linear machine analysis is presented and the effects of saturation are examined as a parameter change in the linear model.

\section{Field Orientation Torque Characteristics}

A direct field orientation drive can be viewed as a controlled-current controlled slip frequency source supplying the induction motor. For field orientation, the flux and torque currents are orthogonal and are related to the stator current by the equation (magnitude relation)

$$
i_{d s}^{2}+i_{q s}^{2}=i_{q d s}^{2} .
$$

Substituting this result in (4) yields

$$
T=\frac{3}{2} \frac{P}{2} \frac{L_{m}^{2}}{L_{r}} i_{d s}^{2}\left[1+\left(\frac{i_{q s}}{i_{d s}}\right)\right]^{2} \frac{s \omega \tau_{r}}{1+\left(s \omega \tau_{r}\right)^{2}} .
$$

With the correct gain in the slip calculator the slip relation in the steady state is

$$
s \omega \tau_{r}=\frac{i_{q s}}{i_{d s}}
$$

which when substituted in (11) yields the desired linear relation between torque and torque command $i_{q s}$. 


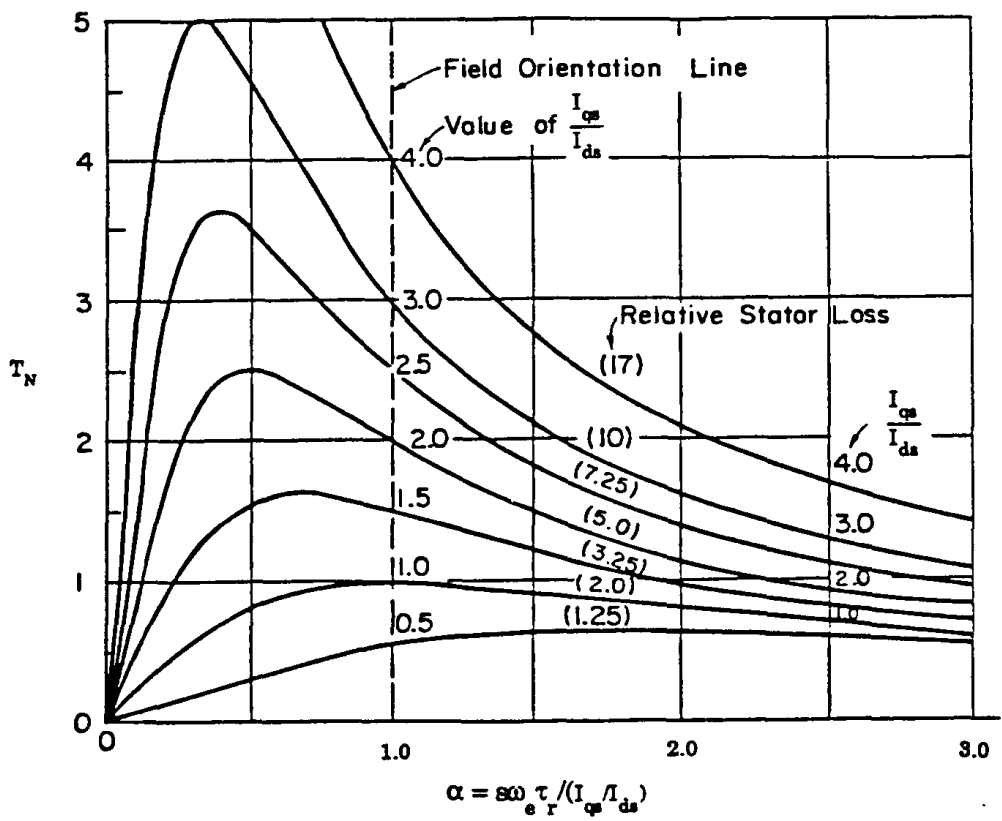

Fig. 9. Field orientation torque characteristics for evaluation of slip gain error sensitivity.

If, however, the slip gain is incorrect such that

$$
s \omega \tau_{r}=\alpha \frac{i_{q s}}{i_{d s}}
$$

the desired cancellation of the nonlinear terms in (11) will not occur and the system exhibits detuned operation.

A very useful set of characteristic torque curves can be obtained by substituting (13) in the torque relation (11) and normalizing the result to yield

$$
T_{N}=\left[1+\left(\frac{i_{q s}}{i_{d s}}\right)^{2}\right] \frac{\alpha \frac{i_{q s}}{i_{d s}}}{1+\left(\alpha \frac{i_{q s}}{i_{d s}}\right)^{2}}
$$

where

$$
T_{N}=\frac{T}{\frac{3}{2} \frac{P}{2} \frac{L_{M}^{2}}{L_{r}} i_{d s}^{2}} .
$$

The normalized torque $T_{N}$ can be interpreted as the torque in per unit based on the field-oriented torque that exists when $i_{q s} /$ $i_{d s}=1.0$.

The resulting torque characteristics are illustrated in Fig. 9, where the normalized torque $T_{N}$ is plotted as a function of the gain error $\alpha$. Since $s \omega \tau_{r}=i_{q s} / i_{d s}$ corresponds to field orientation, the vertical line at $\alpha=1$ represents field-oriented operation. Note that along this line the torque is a linear function of $i_{q s} / i_{d s}$. All other vertical lines represent detuned operation $(\alpha \neq 1)$ and the curves permit easy evaluation of the change in torque resulting from any specific slip gain error.

For example, for a (nonsaturating) machine operating with $i_{q s} / i_{d s}=2.0$ the torque reduction caused by operation with a slip gain error of $100 \%(\alpha=2)$ is $1.2 / 2.0$ or 0.6 . If the error is $-50 \%(\alpha=0.5)$ the detuned torque is $2.5 / 2.0$ or 1.25 times larger than the field-oriented torque.

The curves in Fig. 9 are also labeled to indicate the relative stator $I^{2} R$ loss, which is proportional to $1+\left(i_{g s} / i_{d s}\right)^{2}$. This allows determination of the increase in stator $I^{2} R$ loss caused by detuning. For the example given above, to bring the detuned torque of 1.2 for a $100 \%$ slip gain error back up to 2.0 requires increasing the value of $i_{q s} / i_{d s}$ to nearly 4.0 . This will result in an increase in the stator $I^{2} R$ loss by approximately $17 / 5$, or about a factor of three.

\section{Field Orientation Flux Characteristics}

Detuned operation causes changes in both torque and flux. A set of characteristic curves for the flux changes can be obtained by the same basic approach as for the torque. The resulting normalized flux characteristics are expressed by

$$
\frac{\lambda_{r}}{L_{m} i_{d s}}=\sqrt{\frac{1+\left(i_{q s} / i_{d s}\right)^{2}}{1+\left(\alpha i_{q s} / i_{d s}\right)^{2}}},
$$

and the resulting characteristic curves are illustrated in Fig. 10. Note that positive slip gain errors $(\alpha>1)$ cause high slip and low flux while the converse is true for negative slip gain errors.

\section{General Properties of Detuning}

The characteristic curves of Figs. 9 and 10 are universal and apply to any nonsaturating induction machine. For such machines the sensitivity to detuning is totally dependent on the ratio $i_{q s} / i_{d s}$. Since the curves are universal, they can be used to state general conclusions concerning sensitivity to detuning. 


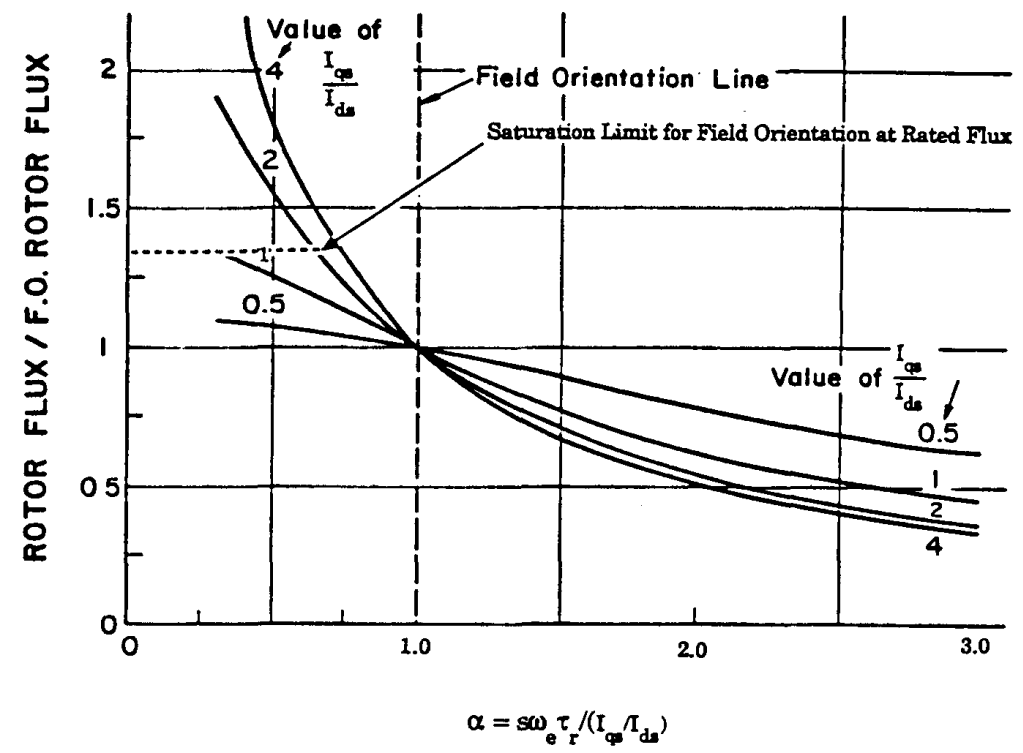

Fig. 10. Field orientation flux characteristics for evaluation of slip gain error sensitivity.

Such conclusions can be applied to compare different machines or the same machine under different operating conditions.

Examination of the curves shows that larger values of the ratio $i_{q s} / i_{d s}$ result in

1) larger torque loss (or gain) for any specific amount of detuning,

2) larger changes in flux for any specific amount of detuning,

3) larger changes in stator $I^{2} R$ loss to recover the normal field-oriented torque.

Clearly, operation at reduced values of $i_{q s} / i_{d s}$ results in much less sensitivity to detuned operation in all respects. One immediate general conclusion is that large machines, which inherently have relatively lower values of magnetizing current, are inherently more sensitive to detuning than small machines. Also, since the magnetizing current for a given frame size increases with the number of poles, high pole number machines are inherently less sensitive to detuning.

\section{Influence of Saturation}

Because of the normalization employed in Figs. 9 and 10 the saturation characteristic of Fig. 3 is not readily incorporated into the analysis. However, qualitatively the saturation can be viewed as reducing the value of the equivalent magnetizing reactance and thus increasing the required magnetizing current. Since this has the effect of reducing the value of $i_{q s} / i_{d s}$ compared to the linear case, it is clear that saturation will always reduce the sensitivity to detuning. To a first approximation the sensitivity of a specific machine can be evaluated by using the chord slope magnetizing reactance shown in Fig. 3 and applying the curves in Figs. 9 and 10.

A very important conclusion based on these results is that the sensitivity to detuning will become much greater for any given machine when it is operated in the field-weakening mode (constant horsepower). The change can be quite dramatic since the value of $i_{q s} / i_{d s}$ can change by a factor of four or more as the machine speed is increased to twice base speed. This is especially true in small machines that normally operate with $i_{q s} / i_{d s}$ near unity and may have very substantial saturation at rated flux. To illustrate this phenomenon, consider a machine which has $i_{o s} / i_{d s}=1.0$ at rated flux and torque and 4.0 at $50 \%$ flux (approximately twice rated speed). The effect of $+100 \%$ detuning at full flux would be a torque reduction to about $0.8 \mathrm{pu}\left(\alpha=2, i_{q s} / i_{d s}=1.0\right.$ on Fig. 9) while at $50 \%$ flux the reduction would be to about $0.5 \mathrm{pu}\left(\alpha=2, i_{q s} / i_{d s}=\right.$ 4.0 on Fig. 9). Note that the torque loss more than doubles under the field-weakened condition.

The influence of the hard saturation of Fig. 3 can be incorporated in Fig. 10 if the curve is viewed as having rated flux at field orientation. In this case the saturation will simply limit the flux for low values of $\alpha$ to the saturation flux as illustrated by the dashed line labeled "saturation limit" in the figure. Note that this implies that high values of $i_{q s} / i_{d s}$ cause a very rapid move to saturated conditions for negative slip gain errors.

\section{Saturation Effects at Constant Flux Command}

Saturation can have an influence on torque linearity and losses even when operating with a constant flux command. This occurs, for example, when the torque command is increased while holding the flux command fixed. The increased current in the stator actually results in higher values of stator and air gap flux, and hence the saturation level, even though the rotor flux command remains constant.

This change in saturation level is, however, to some extent self compensating for the following reason. The increased torque command results in higher air gap flux, more saturation and hence a somewhat lower value of magnetizing reactance. This would tend to cause a small reduction of the rotor flux if the flux command is constant. However, the reduced magnet- 


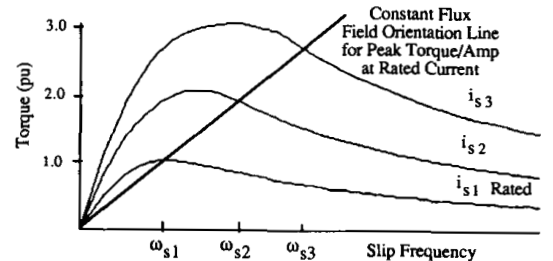

Fig. 11. Determination of flux producing current and slip gain from experimental data.

izing inductance causes a slip gain error which tends to make the slip too small $(\alpha<1)$. Since small slip detuning tends to cause an increase in rotor flux (see Fig. 10), there is a tendency to compensate the flux error. As a result the field orientation torque characteristic tends to remain linear despite the detuning resulting from saturation. This is the basic reason why field orientation systems tend to exhibit good torque linearity even under highly saturated conditions.

\section{Experimental Determination of Flux-Producing Current}

An experimental procedure may be used for determining the flux producing stator current component $i_{d}$. The procedure is especially well suited for cases where signal noise is present because it allows redundant points to be taken to improve convergence to unbiased estimates.

The procedure requires that the field-oriented controller be used as a variable slip converter (either with motor stalled or under load at speed). At least two torque versus slip curves must be acquired as shown in Fig. 11. The data for Fig. 11 is experimental data taken on the motor cited in Appendix II using a field-oriented controller with the machine under stalled conditions. At least one of the curves should be at the rated (or converter limited) value of stator current amplitude.

To determine, for example, the $i_{d}$ component corresponding to the peak torque per ampere at rated current, a constant flux (straight) line from the origin to the peak of the rated current curve should be drawn as shown. The intersection of the line with all other curves will provide current amplitudes $i_{s}$, slip frequencies $\omega_{s}$, and torques for each point. With this data the following equations may be formed:

$$
\begin{aligned}
& i_{s 1}^{2}=i_{d}^{2}+i_{q 1}^{2} \\
& i_{s 2}^{2}=i_{d}^{2}+i_{q 2}^{2}
\end{aligned}
$$

and from the slip gain constraint:

$$
\begin{array}{cc}
\omega_{s 1}=K_{s} i_{q 1} \\
\omega_{s 2}=K_{s} i_{q 2} \\
. & .
\end{array}
$$

These equations may be combined and rewritten as the following matrix equation:

$$
\left[i_{s i}^{2}\right]=i_{d}^{2}+\frac{1}{K_{s}^{2}}\left[\omega_{s i}^{2}\right] .
$$

To solve for $K_{s}$ and $i_{d}$ requires only to perform a linear least-squares regression analysis on the squared values of both $1 / K_{s}$ and $i_{d}$ based on the matrix equation:

$$
\boldsymbol{Y}=\boldsymbol{A}+\boldsymbol{B} \boldsymbol{X}
$$

where $A$ and $B$ are to be estimated given $X$ and $Y$. Note that if only two points are selected, then the four equations allow explicit computation of $K_{s}, i_{d}$, and the corresponding $i_{q}$ 's. However, use of additional data points will improve the accuracy of the estimates as is true for any regression analysis.

\section{Conclusion}

- Magnetic saturation plays an important role in fieldoriented induction machine drives.

- A simple piecewise linear ideal limiter model of saturation provides a useful tool for analysis of torque and flux production in indirect feedforward field-oriented induction machines.

- The model demonstrates the slow increase of rotor flux and thus diminishing electromagnetic torque production due to operation at flux command levels significantly above rated flux.

- Under saturation conditions the peak torque per stator ampere is best produced by increasing the torque producing current command's proportion of the total stator current. This is equivalent to stating that the magnetizing current should not be increased significantly due to its diminishing ability to increase rotor flux.

- Lower relative torque production (lower $T /\left|i_{q s}\right|^{2}$ ) will occur (and thus lower efficiency will result) if the machine is run with flux levels in the saturation region. An optimal flux command exists and is nearly constant over a wide range of torques.

- A set of generalized characteristic curves for detuned operation has been presented. These curves illustrate that the ratio $i_{q s} / i_{d s}$ totally controls the sensitivity to detuning.

- Saturation tends to lower the ratio $i_{q s} / i_{d s}$ and therefore significantly reduces the sensitivity to detuning. Hence, field-weakened operation (constant horsepower) will exhibit greater sensitivity to detuning than normal constant torque operation.

- A simple experimental means of determining the magnetizing current for peak torque per ampere has been presented as a simple nonlinear regression analysis.

\section{APPENDIX I}

\section{Parameter Definitions}

All vector quantities are defined in a reference frame oriented to the rotor flux, and are of the form

$$
\bar{v}_{q d s}=v_{q s}-j v_{d s} .
$$

$\bar{v}_{q d s} \quad$ Stator voltage.

$\bar{i}_{q d s} \quad$ Stator current.

$\bar{i}_{q d r} \quad$ Rotor current.

$\bar{\lambda}_{q d s} \quad$ Stator flux linkage.

$\bar{\lambda}_{q d r} \quad$ Rotor flux linkage.

$L_{m} \quad$ Magnetizing inductance.

$L_{s}, R_{s}$ Stator inductance and resistance. 
$L_{r}, R_{r} \quad$ Rotor inductance and resistance.

$\tau_{r} \quad$ Rotor time constant, $\tau_{r} \equiv L_{r} / R_{r}$

$\omega_{e} \quad$ Excitation frequency.

$\omega_{r} \quad$ Rotor speed, (elec. $\left.\mathrm{rad} / \mathrm{s}\right)$.

$\omega_{s} \quad$ Slip frequency, $\omega_{s} \equiv \omega_{e}-\omega_{r}$.

$p \quad$ Time derivative operator.

$P \quad$ Number of poles.

$T \quad$ Electromagnetic torque.

APPENDIX II

Machine Parameters Used in Illustrations (Test Values)

$\begin{array}{ll}L_{m} & 1.213 \mathrm{pu} \\ L_{s} & 1.268 \mathrm{pu} \\ L_{r} & 1.242 \mathrm{pu} \\ R_{s} & 0.0497 \mathrm{pu} \\ R_{r} & 0.0323 \mathrm{pu} \\ Z_{\text {base }} & 23.646 \Omega @ 60 \mathrm{~Hz}, 230 \mathrm{~V} \\ I_{\text {base }} & 5.616 \mathrm{A@} @ 60 \mathrm{~Hz}, 230 \mathrm{~V} .\end{array}$

\section{Motor Nameplate Values}

$3 / 0 \mathrm{hp}, 3 \varnothing$ wye-connected

$230 / 460 \mathrm{~V}$

$8.4 / 4.2 \mathrm{~A}$

4 poles.

\section{ACKNOWLEDGMENT}

The authors wish to acknowledge the financial support of the Wisconsin Electric Machines and Power Electronics Consortium (WEMPEC).

\section{REFERENCES}

[1] F. Khater, R. D. Lorenz, D. W. Novotny, and K. Tang, "The selection of flux level in field-oriented induction controllers with consideration of magnetic saturation effects," IEEE Trans. Ind. Appl., Mar./Apr. 1987.

[2] J. M. Loehrke and R. D. Lorenz, "Torque characteristics of fieldoriented induction machines," in Conf. Rec., 1985 Conf. on Appl. Motion Control, pp. 101-107.

[3] D. W. Novotny and R. D. Lorenz, (Eds.), "Introduction to field orientation and high performance ac drives," tutorial book, 1986 IEEE/IAS Annual Meeting, Denver, CO, Sept. 28-Oct. 3.

[4] H. C. J. de Jong, "Saturation in electrical machines," in Proc. International Conference on Electrical Machines, Part 3, Athens, Greece, Sept. 15-17, 1980, pp. 1545-1552

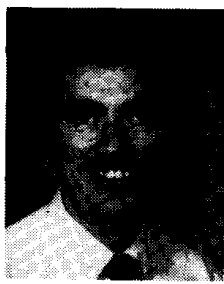

Robert D. Lorenz (S'83-M'84) received the B.S., M.S., and Ph.D. degrees in mechanical engineering from the University of Wisconsin-Madison, in 1969,1970 , and 1984 respectively.

Since 1984 he has been a member of the faculty of the University of Wisconsin-Madison, where he is an Associate Professor of Mechanical Engineering and Affiliate Associate Professor of Electrical and Computer Engineering. In this position he act as Associate Director of the Wisconsin Electric Machines and Power Electronics Consortium and as Co-Director of the Advanced Automation and Robotics Consortium. He was a Visiting Research Professor in the Electrical Drives Group of the Catholic University of Leuven, Leuven, Belgium and in the Electrical Drives Institute of the Technical University of Aachen, West Germany in the Summers of 1989 and 1987, respectively. In 1969-1970 he did research at the Technical University of Aachen, West Germany. From 1972 to 1982 he was a member of the research staff at the Gleason Works, in Rochester, NY. His current research interests include optical and electromagnetic sensor technologies, real-time digital signal processing techniques, electromagnetic actuator design and control, and ac drive and high precision machine control technologies.

Dr. Lorenz is a member of the IAS Industrial Drives Committee, the Machine Tools, Robotics, and Factory Automation Committee, and the Industrial Control Committee. He is an active consultant to many organizations and is a Registered Professional Engineer in the States of New York and Wisconsin. He is a member of ASME, ISA, and SPIE.

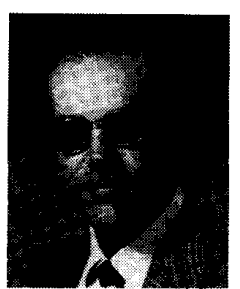

Donald W. Novotny (M'62-SM'77-F'87) received the B.S. and M.S. degrees in electrical engineering from the Illinois Institute of Technology, Chicago, in 1956 and 1957, respectively, and the Ph.D. degree from the University of WisconsinMadison in 1961 .

Since 1961 he has been a member of the faculty at the University of Wisconsin-Madison, where he is currently Professor and Co-Director of the Wisconsin Electric Machines and Power Electronics Consortium (WEMPEC). He served as Chairman of the

Electrical and Computer Engineering Department from 1976 to 1980 and as an Associate Director of the University-Industry Research Program from 1972 to 1974 and from 1980 to the present. He has been active as a consultant to many organizations and a Visting Professor at Montana State University, Boseman, the Technical University of Eindhoven, Eindhoven, The Netherlands, the Catholic University of Leuven, Leuven, Belgium, and a Fullbright Lecturer at the University of Ghent, Ghent, Belgium. His teaching and research interests include electric machines, variable frequency drive systems, and power electronic control of industrial systems.

Dr. Novotny is a member of ASEE, Sigma XI, Eta Kappa Nu, and Tau Beta $\mathrm{Pi}$, and is a Registered Professional Engineer in the State of Wisconsin. 